\title{
La teoria del diritto come pratica sociale e la coercizione
}

\author{
The Theory of Law as Social Practice and Coercion
}

Francesco VIOLA

Emérito de Filosofia del derecho

Università di Palermo (Italia)

https://orcid.org/0000-0001-7004-6635

francesco.viola@unipa.it

Abstract: Poiché non si può trattare del posto che la coercizione ha nel concetto di diritto senza far riferimento ad una specifica teoria del diritto, il paper ricostruisce le linee generali della teoria del diritto come pratica sociale, focalizzando in particolare l'obiettivo del diritto di custodire l'agency dei consociati per contrastare l'uso arbitrario del potere. Nella seconda parte si affronta dal punto di vista del diritto come pratica sociale il tema generale della coercizione giuridica e ci si chiede soprattutto se l'uso giuridico della forza non sia anch'esso nella sostanza una forma di potere che contraddice il fine generale della custodia dell'agency.

Parole-chiave: teoria del diritto; coercizione; diritto come pratica sociale; ragion pratica; normatività del diritto; rule of law.
Abstract: It is impossible to face the role of coercion in the concept of law without reference to a specific theory of law. Then, in the first part, the paper elaborates the general lines of a theory of law as a social practice, focussed precisely on the aim of protecting agency against arbitrary power. In the second part, the focus is legal coercion. The main question is whether the legal use of force is another form of power that contrast that general goal of protecting agency or not.

Keywords: legal theory; coercion; law as social practice; practical reason; normativity of law; rule of law.

a questione del posto che la coercizione ha nel concetto di diritto è una spina nel fianco di ogni teoria e filosofia giuridica. La varietà delle risposte è tale da scoraggiare qualsiasi tentativo di una soluzione soddisfacente. La dottrina tradizionale vede nella coercizione uno degli elementi essenziali del diritto in quanto ne rafforza l'efficacia, che a sua volta è necessaria poiché un diritto inefficace serve a ben poco. Quest'argomento è sembrato anche decisivo per ben separare il diritto dalla morale e, conseguentemente, la coercizione ha assunto un rilievo sempre maggiore sino ad identificarsi con lo stesso concetto di diritto. Tuttavia nella teoria giuridica del nostro tempo, al contrario, si può chiaramente notare una forte tendenza a sottodeterminare il ruolo della coercizione. Ciò è favorito tra l'altro dall'ascesa del diritto internazionale e dalla globalizzazione, che hanno separato il luogo della produzione del diritto, non più monopolio dello Stato, dal luogo dell'esercizio della forza pubblica, di cui 
lo Stato resta ancor oggi il principale titolare. La normatività del diritto sembra dipendere sempre meno dalla coercizione, sino al punto da potersi pensare un concetto di diritto privo della coercizione come suo elemento essenziale, come sarebbe ipoteticamente quello proprio di una società di angeli o di santi.

A questo punto si può dire che tutta la gamma dei possibili rapporti tra diritto e coercizione sia stata esplorata. Per alcuni la coercizione è così essenziale per il diritto da coprire tutto il suo concetto (Bentham, Austin, Kelsen). Per altri è essenziale, ma è ben lungi dall'essere sufficiente a definire il diritto (Dworkin, Finnis). Per alcuni non c'è alcuna connessione necessaria tra normatività giuridica e coercizione (Hart, Raz, Shapiro). Per altri si può parlare solo di un'attitudine potenziale del diritto ad essere imposto con la forza (Lamond, Green). Per alcuni è essenziale al diritto l'autorizzazione all'uso della forza nel caso di una violazione di almeno alcune norme giuridiche (Himma). Ed infine, per altri ancora la coercizione, pur non essendo un carattere essenziale del diritto, è presente di fatto in modo pervasivo nella pratica giuridica e di questo la teoria del diritto deve tener conto (Schauer) ${ }^{1}$.

Non intendiamo entrare in questa discussione che non ha trovato ancora il suo assetto definitivo, come d'altronde è proprio di tutte le discussioni filosofiche.

Qui ci si chiede come la concezione del diritto come pratica sociale dovrebbe affrontare il tema della coercizione. Questo interrogativo ha due obiettivi: quello generale è volto a rafforzare la pretesa della concezione del diritto come pratica sociale di tradursi in una vera e propria teoria del diritto ${ }^{2}$, fugando l'impressione di vaghezza e genericità che l'accompagnano, e quindi, in particolare, e questo è il secondo obiettivo, di render conto da questo punto di vista anche del concetto di coercizione giuridica ${ }^{3}$.

Preliminarmente occorre precisare che la coercizione può essere intesa in senso lato o in senso stretto. In senso lato è il modo di esercitare pressioni sulla

1 Per una sintesi di queste diverse posizioni teoriche e per i riferimenti bibliografici rinvio a HIMMA, K.E., «Law and Coercion», in M. Sellers e S. Kirste (eds.), Encyclopedia of Law and Social Philosophy, Springer, Dordrecht, 2017.

2 Preciso che non considero possibile elaborare una teoria del diritto senza presupposti e assunzioni filosofiche. L'ideale di una teoria del diritto come pura scienza, al modo delle scienze naturali, non è altro che un wishful thinking. Cfr. VIOLA, F., «La filosofia del diritto come pratica sociale», relazione presentata nel I Congreso de filosofía del derecho para el mundo latino (Alicante, 26-28 maggio 2016).

3 Proseguo qui il discorso iniziato nel mio Il diritto come pratica sociale, Jaca Book, Milano, 1990, che, pertanto, per molti versi presuppongo. 
volontà altrui mediante minacce, sì da indurre qualcuno a fare ciò che non vorrebbe fare, o che potrebbe non fare se tali minacce mancassero, e che è deliberatamente rivolto a questo scopo ${ }^{4}$. In tal senso nel campo giuridico è sinonimo di «sanzione» intesa genericamente sia come minaccia di conseguenze sfavorevoli nel caso di violazione di una norma giuridica, sia come risposta o reazione in seguito all'avvenuta violazione $e^{5}$. In senso stretto si ha coercizione o coazione in presenza della minaccia o dell'uso della forza fisica, cioè con atti violenti. E sono soprattutto le sanzioni giuridiche coattive che terremo qui presenti, perché sono quelle che creano più difficoltà per una teoria del diritto come pratica sociale.

\section{Pratiche SOCIALI IN SENSO LARGO E IN SENSO STRETTO}

Che il diritto sia una pratica sociale è una constatazione lapalissiana e proprio per questo generica e ben poco significativa. Cercare di dare ad essa una dignità scientifica sembra un'impresa disperata.

Le pratiche sociali non sono una categoria ben definita, ma sono molto eterogenee fra loro per l'oggetto, i processi di azione e la configurazione delle regole. In generale esse designano attività congiunte di una pluralità di persone che partecipano in qualche modo a uno stesso corso d'azione. Ma la comunanza tra le differenti pratiche sociali si ferma qui e ciò è ancora ben poco. La vita sociale è popolata da una miriade di pratiche sociali in cui si esprime nel modo più complesso e articolato la relazionalità umana e la varietà dei suoi fini. È forte la tentazione di cercare il concetto di diritto non già nel suo essere una pratica sociale, ma altrove. $\grave{E}$ possibile configurare il diritto in quanto pratica sociale come una teoria giuridica? Qui cercherò soltanto di esplorare alcune condizioni di questa possibilità.

Innanzi tutto bisognerà distinguere una concezione allargata da una concezione ristretta di pratica sociale.

4 Questa definizione di coercizione, che adombra quella di Nozick, punta sul destinatario delle minacce più che sul loro autore o sulla loro funzione e questo è stato considerato un difetto. Cfr. NozicK, R., «Coercion», in Philosophy, Science, and Method: Essays in Honor of Ernest Nagel, S. Morgenbesser et al. (eds.), St. Martin's Press, New York, 1969, pp. 440-472. Per le critiche da posizioni opposte cfr. BERMAN, M.N., «The Normative Functions of Coercion Claims», Legal Theory, 8 (2002), pp. 45-89; e ANDERSON, S.A., «The Enforcement Approach to Coercion», Fournal of Ethics \& Social Philosophy, 5 (2010), 1, pp. 1-31.

5 Per il concetto di sanzione giuridica rinvio a BoBbIO, N., «Sanzione» (1969), ora in ID., Contributi ad un dizionario giuridico, Giappichelli, Torino, 1994, pp. 307-333. 
Secondo la concezione allargata qualsiasi forma di attività cooperativa mediante cui due o più persone intendono compiere un'impresa comune deve essere considerata una pratica sociale ${ }^{6}$. Secondo la visione più ristretta una vera e propria pratica è «sociale» in senso proprio sia per la sua ampia diffusione in un contesto sociale determinato, sia in quanto riguarda beni o obiettivi sociali di una qualche rilevanza significativa. Così cucinare insieme o organizzare un'escursione di gruppo è una pratica sociale per la concezione allargata, ma non lo è per la concezione ristretta. L'esempio più emblematico per quest'ultima è senz'altro il linguaggio, ma lo sono anche le scienze e le arti, i costumi sociali o le mode e persino l'etichetta.

La comprensione di queste attività in quanto tali esige, per non vanificare la loro dimensione operativa, l'abbandono di un approccio essenzialistico, volto a risolverle in qualche loro componente come caratterizzante ${ }^{7}$. La connessione dei fatti sociali e linguistici, in cui una pratica consiste, si coglie solo nella prospettiva dell'azione da compiere. Una pratica si comprende solo dal punto di vista pratico, cioè guardando alle giustificazioni e alle ragioni per compiere un'azione o astenersi da essa. Dobbiamo guardare all'uso e alla sua evoluzione interna.

Come nota Dworkin nel noto esempio della pratica della cortesia, dapprima la si segue in modo irriflesso, poi si comprende la sua finalità interna e, infine, ci si chiede se questa non possa essere meglio raggiunta mediante interpretazioni differenti delle sue regole o mediante un loro mutamento. Di conseguenza l'usanza stessa si evolve, pur nella convinzione che ogni fase si basi sull'interpretazione di quella precedente ${ }^{8}$. Mutamento e continuità contribuiscono insieme a mantenere in vita una pratica sociale e a custodirne l'identità. Un cambiamento, anche creativo, è giustificato solo dall'esigenza di restare fedeli alla ragion d'essere profonda della pratica, ai valori che essa intende perseguire e ai fini che è volta a raggiungere.

Se il diritto è una pratica sociale, lo è secondo la visione ristretta o esigente, ed allora bisogna cercare d'individuare quali condizioni questa debba

6 Nella concezione allargata viene focalizzata come fondamentale il problema della condivisione dell'intenzione (shared intention). Cfr. Bratman, M., Faces of Intention, Cambridge University Press, Cambridge, 1999, pp. 94-95.

7 Sulla distinzione di Dworkin tra teorie semantiche e teorie interpretative del diritto cfr. VIOLA, F.; ZACCARIA, G., Diritto e interpretazione. Lineamenti di teoria ermeneutica del diritto, IX ed., Laterza, Roma-Bari, 2016, p. 20.

8 Dworkin, R., Law's Empire, Fontana Press, London, 1986, pp. 47-49. 
rispettare. Non ci si deve aspettare il ricorso a criteri ben precisi e tassativi, perché così si ritornerebbe alla visione essenzialistica del diritto. Si deve andare alla ricerca di una tipologia che si può realizzare secondo una variabile gradualità.

Abbiamo già accennato al criterio della diffusione della pratica in un contesto sociale determinato, cioè al coinvolgimento di un elevato numero di partecipanti (ma quanto elevato?). Tuttavia questo è un criterio meramente quantitativo che di per sé sarebbe poco significativo e che deve essere coniugato con il criterio d'identificazione dei partecipanti. Chi deve essere considerato un «partecipante»? È una domanda a cui non è facile rispondere quando sono coinvolte a diverso titolo numerose persone, com'è proprio nelle imprese cooperative complesse. Ma soprattutto la risposta dipende dalla natura della pratica in questione. Come vedremo, questo punto è particolarmente interessante per la definizione della pratica giuridica.

Una pratica sociale in senso stretto deve anche avere un grado elevato di stabilità e durata. Ciò non significa - come già s'è detto - che non sia mutevole al suo interno anche in modo rilevante. Al contrario quanto più è durevole, tanto più è mutevole nei modi e nelle forme di partecipazione ad essa, pur restando se stessa, come avviene per il linguaggio. Ciò non significa neppure che non possa estinguersi, come avviene per le lingue che appunto chiamiamo «morte». Non solo gli esseri umani, ma anche tutte le opere umane sono mortali, anche quelle compiute in comune. Gli ordinamenti giuridici nascono e muoiono, ma sono tali in quanto esibiscono e promettono stabilità e durata, cioè una certa qual longevità. Queste caratteristiche alimentano la fiducia tra i partecipanti, l'affidamento alle regole comuni, lo spirito di cooperazione, cioè rafforzano la socialità umana. Persino le rivoluzioni, che sono transitorie per definizione, hanno a volte tentato di presentarsi come permanenti, come Trotsky pensava che dovesse essere la rivoluzione comunista.

Partecipazione diffusa e stabilità sono gli aspetti esteriori necessari di una pratica sociale intesa in senso stretto, ma la sua descrizione ed identificazione richiede una risposta a due quesiti fondamentali: quello riguardante a quali condizioni si agisce insieme (o quando un'azione è compiuta insieme ad altri, cioè è un'azione congiunta o comune a più persone) e quello riguardante ciò che si fa insieme (o l'opera di cui si tratta o il contenuto dell'azione congiunta o lo scopo a cui è diretto l'agire insieme). È importante affrontare entrambe le questioni se vogliamo identificare una pratica sociale determinata, qual è, ad esempio, quella del diritto, altrimenti non riusciremo mai a diradare le nebbie che avvolgono questo concetto. Non basta rispondere alla questione del come, 
ma bisogna affrontare anche la questione del cosa, che molto spesso resta inevasa perché erroneamente ritenuta irrilevante.

Le concezioni analitiche di pratica sociale, che hanno tratto ispirazione dalla versione convenzionalistica di Lewis ${ }^{9}$ e da quella cooperativa di Brat$\operatorname{man}^{10}$, hanno focalizzato in modo esclusivo la questione del come. Infatti, per questa prospettiva filosofica, ispirata all'individualismo metodologico, la sfida principale è quella di spiegare come un'azione possa essere compiuta da più soggetti separati in modo che si realizzi un'unità sistematica delle intenzioni individuali ${ }^{11}$. I risultati di queste indagini sono stati senza dubbio illuminanti per lo studio delle convenzioni e delle relazioni tra le intenzioni dei partecipanti ad un'attività condivisa (shared cooperative activity). Ma né Lewis né Bratman hanno avuto come specifico loro oggetto di studio il diritto o il tipo particolare di pratiche a cui esso appartiene. La loro prospettiva era quella di elaborare una concezione in grado di applicarsi a tutte le forme d'interazione nelle attività comuni, cioè quella che abbiamo definito come una concezione allargata di pratica sociale. Anzi Bratman ha esplicitamente evitato di prendere in considerazione forme d'interazione di carattere autoritativo. E si capisce bene il perché. La presenza di un'autorità introduce necessariamente la dimensione normativa (e anche la legittimità della coercizione), ma questa a sua volta minaccia il carattere cooperativo della partecipazione intesa come agire strategico, cioè libero da vincoli normativi ${ }^{12}$.

\section{MODELLI DI PRATICA GIURIDICA}

Siccome il diritto è una di quelle pratiche sociali che richiedono la presenza dell'autorità, ci si chiede se il modello di Bratman possa sopportare senza contraddizioni di essere integrato in tal senso. Il risultato inevitabile dei tentativi già fatti conduce a restringere l'ambito dei partecipanti alla pratica giuridica ai funzionari pubblici, che creano e applicano regole primarie

9 LewIs, D., Convention: A Philosophical Study, Harvard University Press, Cambridge, 1969.

10 V. supra n. 6.

11 Cfr. Smith, M.N., «The Law as A Social Practice: Are Shared Activities at the Foundations of Law?», Legal Theory, 12 (2006), p. 269.

12 Per la differenza tra interazione strategica e interdipendenza normativa rinvio a PASTORE, B., et al., Le ragioni del diritto, Il Mulino, Bologna, 2017, pp. 41-55. 
di condotta nel rispetto di regole secondarie di competenza, come insegna il modello hartiano ${ }^{13}$. Questi soggetti normalmente non agiscono per il timore delle pene né sono mossi dall'interesse di aggirare il diritto quando possono farlo impunemente, pur essendo sottoposti alla legge e alle autorità superiori. Ma è questo il modo migliore di descrivere una pratica giuridica? Non è forse essa il luogo dell'incontro e dello scontro di una ben più ampia varietà di soggetti, di interessi e di interpretazioni relative a ciò che debba intendersi come diritto? La teoria del diritto come pratica sociale non accetta di ridurre la complessità di ciò che è orientato all'azione in nome di una sterile scientificità che conosce senza comprendere.

Come già s'è detto, l'identificazione dei partecipanti è una spia rivelativa del modo d'intendere la pratica giuridica. Possiamo considerare come un modello istituzionale di pratica giuridica quello per cui i veri e propri partecipanti sarebbero i funzionari pubblici. Ma ciò è controintuitivo, anche accettando una considerazione molto allargata di questi soggetti, cioè comprendente anche gli avvocati, i giuristi e i burocrati e tutti coloro che esercitano una qualche funzione pubblica (notai, cancellieri, poliziotti, etc.) $)^{14}$. I grandi esclusi sarebbero, infatti, i cittadini, cioè proprio coloro le cui azioni sono coordinate dal diritto. Anche se l'opera propria del diritto fosse la produzione di norme, non v'è alcun dubbio che gli stessi destinatari del diritto in realtà partecipano alla sua produzione, e non solo mediante le loro attività private e mediante i loro diritti politici nei regimi democratici, ma soprattutto nella stessa obbedienza alle norme che contribuisce a svilupparne, determinarne, e a volte a modificarne profondamente il significato originario ${ }^{15}$. La questione dell'obbedienza alle norme giuridiche è parte integrante di una teoria del diritto.

Si può discutere se gli spettatori facciano parte della rappresentazione di un'opera teatrale o dell'esecuzione di un brano musicale. Si può legittimamente dubitare che i tifosi facciano parte del gioco del calcio inteso come pratica sociale. Ma che i cittadini, ancor più dei funzionari, debbano

13 Per l'uso in tal senso del modello di Bratman cfr. SHAPIRO, S. J., «Law, Plan and Practical Reason», Legal Theory, 8 (2002), p. 418 e anche Coleman, J., Practice of Principle. In Defence of a Pragmatist Approach to Legal Theory, Oxford University Press, Oxford, 2001, pp. 95-102. Coleman in seguito ha rinunciato a servirsi di questa prospettiva per la sua teoria del diritto.

14 SMITH, p. 272.

15 Cfr. le acute osservazioni a proposito di Cover, R.M., «Nomos and Narrative», Harvard Law Review, 97 (1983), 1, pp. 4-68. 
essere considerati come attori della pratica giuridica, non dovrebbe essere messo in dubbio, poiché non solo la loro azione, come quella dei funzionari, contribuisce a dar forma all'impresa comune del diritto, ma anche perché il fine del diritto riguarda proprio loro. Considerarli come beneficiari passivi di un'autorità, anche se intesa come servizio, sarebbe una forma di paternalismo giuridico.

Questa visione meramente procedurale della pratica giuridica non è soddisfacente da tanti punti di vista il cui esame è molto istruttivo.

Senza dubbio il diritto è una prassi sociale istituzionalizzata, non occasionale e intenzionale. Questa pratica sociale, che contiene istituzioni, è in se stessa un'istituzione? La risposta dipende dal concetto di istituzione, che ha un carattere stipulativo. Se riteniamo che un'istituzione sia per definizione a servizio di valori e obiettivi esterni ad essa ${ }^{16}$, allora la pratica giuridica non è un'istituzione in quanto porta in se stessa i valori che la giustificano. Ma, se invece adottiamo la distinzione tra una procedura e un'istituzione, allora le cose cambiano.

Secondo Habermas, ad esempio, si tratta di due modi diversi di legittimare le norme giuridiche ${ }^{17}$. Il diritto come medium, cioè come procedura, sottopone ad una giuridificazione gli stati di fatto sociali mediante processi di positività, generalità e formalità. Questa coltre formale copre - secondo Habermas - la realtà sociale (i mondi vitali), la nasconde e progressivamente la fagocita. Le norme sono giustificate e legittimate dagli stessi procedimenti che le generano. Le procedure colonizzano le istituzioni sociali. Il diritto come istituzione nel senso habermasiano ${ }^{18}$, invece, si presenta come un insieme di procedimenti di regolazione del conflitto che sono adeguati alle strutture dell'agire orientato all'intendersi: processi di formazione discorsiva della volontà e procedimenti di dibattito e decisione orientati al consenso. In tal mondo mantiene i suoi legami con la componente sociale del mondo vitale. Questo è, dunque, un modello sociale di pratica giuridica ${ }^{19}$.

16 Questo è il concetto di istituzione secondo MacIntyre, A., After Virtue. A Study in Moral Theory, III ed., University of Notre Dame Press, Notre Dame (Indiana), 2007, p. 194.

17 Cfr. Habermas, J., Teoria dell'agire comunicativo, trad. di P. Rinaudo, II, Il Mulino, Bologna, 1986, p. 775 e ss.

18 Lo stesso potrebbe dirsi per l'istituzionalismo di Maurice Hauriou e per il neoistituzionalismo di Neil MacCormick.

19 Cfr. Viola, F., «Pratiche sociali: istituzioni e procedure», Rivista di studi utopici, 2 (2007), 3, pp. 97-106. 
La procedura è una regola tecnica nel senso che la sua applicazione produce immancabilmente il risultato voluto. Un'istituzione in senso habermasiano, invece, pur contenendo al suo interno delle procedure, è una forma di vita sociale, in cui comportamenti e regole sono fusi insieme, costituendo una struttura tipica diretta a realizzare azioni comuni all'interno di determinati contesti sociali. La pratica sociale appartiene al mondo dell'agire, mentre la procedura appartiene al mondo del fare. Una pratica sociale è una struttura sociale dell'agire che porta in se stessa la propria giustificazione ${ }^{20}$.

Come si può notare, questo approccio, a differenza di quello analitico a cui sopra s'è accennato, ha presente non solo la questione del come, ma anche quella del cosa. In una pratica sociale ci sono beni da realizzare, valori da perseguire, fini da raggiungere che sono «interni» alla pratica. Ciò significa che si realizzano, si perseguono o si raggiungono nel fatto stesso dell'agire insieme. Qui siamo nel punto di contatto tra agire e fare, poiché le azioni intersoggettive producono un'opera esteriore. Ma non si tratta di operazioni, il cui scopo è conseguire un risultato concreto. Si tratta di prestazioni, che sono definite dal modo corretto di eseguirle e in questo raggiungono il loro fine ${ }^{21}$. Il fine della danza è danzare bene e quella del nuoto è nuotare bene. Per costruire qualcosa bisogna compiere una serie di operazioni, ma il linguaggio o il ragionamento si concretizzano in una serie di prestazioni, che sono governate da regole del ben operare studiate dalla grammatica o dalla logica, cosicché noi possiamo distinguere un modo corretto di parlare o di ragionare da un modo non corretto.

Il diritto positivo appartiene al campo delle prestazioni e queste sono di diversa natura (prestazioni intellettuali e comportamenti sociali). Esso è insieme ed inscindibilmente un complesso di strutture istituzionali, governate da regole interne, e un insieme di pratiche sociali interpretative e argomenta-

20 Qui ovviamente mi riferisco alla ben nota definizione di A. MacIntyre, p. 187), che qui ricordo: «By a 'practice' I am going to mean any coherent and complex form of socially established cooperative human activity through which goods internal to that form of activity are realized in the course of trying to achieve those standards of excellence which are appropriate to, and partially definitive of, that form of activity, with the result that human powers to achieve excellence, and human conceptions of the ends and goods involved, are systematically extended».

21 AGAZZI, E., «Per una riconduzione della razionalità tecnologica entro l'ambito della razionalità pratica», in Galvan, S. (a cura di), Forme di razionalità pratica, Franco Angeli, Milano, 1992, p. 21. Cfr. anche VIOLA, F., «Il diritto tra arte ed etica», in C. Hermida, J. A. Santos (coord.), Una filosofía del derecho en acción. Homenaje al profesor Andrés Ollero, Congreso de los Diputados, Madrid, 2015, pp. 3-17. 
tive, che rendono operative quelle strutture nei contesti sociali. Certamente le procedure sono ben presenti, ma hanno solo un carattere funzionale.

Ora il problema centrale non è più quello di spiegare come possa darsi un'unità sistematica di attività individuali mediante i processi di correferenzialità ed interreferenzialità. $\mathrm{Al}$ contrario si tratta di mostrare che l'agire in comune è originario, in quanto la stessa azione individuale trova in esso il suo senso e la sua misura. La stessa pratica sociale è lo schema interpretativo delle azioni dei suoi partecipanti. Certamente costoro devono voler parteciparvi, ma, a parte il fatto che vi sono pratiche sociali a cui non ci si può sottrarre (e il diritto è una di queste), non è in loro potere farlo in qualsiasi modo e con qualsiasi fine se vogliono parteciparvi correttamente. Insomma, dall'identificazione di una pratica sociale si deduce quale deve essere il ruolo, la funzione e il comportamento dei partecipanti ad essa. Il percorso è non solo esattamente l'opposto della teoria convenzionalistica, ma anche non di tipo descrittivo ma normativo.

$\mathrm{Ci}$ sono, dunque, ragioni per cui una pratica sociale si costituisce e si consolida. Queste ragioni hanno un carattere valutativo e conferiscono normatività alle regole e alle attività che popolano una pratica sociale.

Queste ragioni e finalità hanno un elevato grado di rilevanza sul piano della vita sociale, sicché si possono ritrovare sotto qualche forma o modalità, in modo palese o implicito, in un grado elementare o più sviluppato, in tutte le società umane. Le pratiche sociali animate da ragioni simili hanno tutte un'aria di famiglia, anche se possono differire tra loro profondamente nei valori perseguiti e nei modi di perseguirli.

\section{COMPLESSITÀ DELLE PRATICHE SOCIALI}

È facile constatare che le ragioni per cui prende forma una pratica sociale di grande rilievo non sono mai esclusive, cioè sono presenti in qualche modo anche in altre pratiche sociali. Ad esempio, se si sostiene che il diritto esiste per il fine di coordinare le azioni sociali, si dice senza dubbio cosa esatta, ma lo stesso si potrebbe dire per la politica o per l'economia. Ed allora bisogna chiedersi in che modo il diritto persegue questa finalità, perché è per questo che si distingue dalla politica o dall'economia. Di conseguenza, la questione del cosa si lega alla questione del come. Sono due interrogativi a cui non si può rispondere separatamente, come d'altronde si addice ad una dimensione pratica in cui il fine è specificato dai modi di attuarlo. Per questo è improprio considerare questi ultimi come «mezzi», in quanto non sono estrinseci al fine 
ma una sua interpretazione e concretizzazione ${ }^{22}$. La specificità del cosa è il risultato delle modalità del come.

La comunanza nel fine implica un'interconnessione tra pratiche sociali, sicché l'una trova in un'altra la sua origine o la sua giustificazione, è parassitaria di un'altra e, a volte, cerca di rimediare ai difetti di questa. Apel ha giustamente rimproverato a Wittgenstein di non essersi sufficientemente interrogato su un tipo particolare di giochi linguistici, quelli che si rapportano ermeneuticamente ad altri giochi di linguaggio. Egli pensa non solo alla narrazione di una storia già vissuta o trasmessa dalla tradizione e all'interpretazione di un testo, ma anche a tutte le attività in cui la comprensione ermeneutica trova la sua applicazione: la predicazione, la lezione, la decisione giudiziaria, l'esecuzione di un pezzo musicale ${ }^{23}$.

Le pratiche sociali sono fluide e porose. Di conseguenza, per coglierne la specificità non bisogna perdere di vista $\mathrm{i}$ contesti in cui operano, che a loro volta sono popolati da altre pratiche sociali da cui non possono essere separate. Insomma, la separazione è il modo peggiore di cercare di comprendere una pratica sociale, ma anche la connessione può indurre a perderne di vista la specificità. Dunque, né separazione né connessione, ma distinzione. Questo è fondamentale per comprendere i rapporti del diritto con la politica, la morale e l'economia.

Inoltre, c'è da aggiungere che una pratica sociale ha un carattere riflessivo, com'è evidente nell'esempio dworkiano della cortesia. Ciò significa che ogni pratica sociale è alla ricerca di se stessa, della sua ratio e del modo di realizzarsi al meglio di sé. Ogni azione giuridica è un tentativo di risposta a cosa è il diritto. Ciò avviene mediante attività interpretative e argomentative, che con ciò stesso diventano parte della pratica stessa, dando luogo ad un gioco sempre più ampio e complesso.

Ferma restando l'universalità del principio cooperativo, le pratiche sociali, come le scatole cinesi, possono trovarsi l'una dentro l'altra e in tal modo formare una rete complessa non solo di convenzioni, di presupposizioni e di aspettative, ma anche di significati e di valori. Ciò è particolarmente evidente in quelle pratiche interpretative che ben conoscono il fenomeno dei significati sommersi o impliciti ed il diritto è una di queste.

22 Infatti, più correttamente, Tommaso d'Aquino li indica come «ea quae sunt ad finem». La volontà vuole necessariamente il fine e attraverso questo anche ciò che conduce ad esso, ma che è specificato dalla ragione. Questa è l'opera propria della virtù della prudenza, che presiede alla deliberazione e alla scelta. Cfr., ad esempio, Sum. Theol., I-II, 8, 2 e 57, 5.

23 APEL, K.-O., Trasformation der Philosophie, Suhrkamp, Frankfurt, vol. I, 1973, pp. 368 ss. 
Nel diritto la complicazione ulteriore è data dalla moltiplicazione dei contesti d'interazione. Il primo è orizzontale, perché i partecipanti si considerano come eguali. Il secondo è verticale in quanto riguarda il rapporto tra le autorità e i cittadini. Il terzo riguarda il rapporto tra le autorità stesse e i funzionari. Lon Fuller ha mostrato in modo convincente che la stessa legge è il prodotto di un'interazione di orientamenti finalistici tra i cittadini e il loro governo (vertical interaction thesis). In più è necessaria per la stessa esistenza della legge un certo grado di congruenza tra la legge emanata, le pratiche di sfondo informali e le convenzioni orizzontali tra i cittadini (congruence thesis) ${ }^{24}$. Per questo la contrapposizione fra legge e consuetudine è in linea di principio erronea, poiché non solo una legge tende a produrre una consuetudine senza la quale perderebbe di efficacia, ma anche le consuetudini esistenti contribuiscono a dar forma ai significati della legge. Se una legge si pone in radicale contrasto con una consuetudine radicata, non di rado è quest'ultima ad avere la meglio.

\section{UNA TEORIA GIURIDICA EX PARTE POPULI}

Se ora ci chiediamo in che senso possiamo e dobbiamo annoverare il diritto tra le pratiche sociali in senso stretto, la prima cosa da fare è cercare il fine per cui v'è il diritto. Infatti si agisce sempre per un fine, almeno così fanno le persone ragionevoli, e ciò vale anche per le azioni comuni e per le opere dell'artificio umano.

Quali sono allora i caratteri distintivi del diritto come pratica sociale? Qual è la ratio del diritto?

A domande del genere non si può rispondere in modo univoco. Una pratica sociale di grande rilievo si costituisce per molteplici fini e ha di mira il perseguimento di molteplici valori. Il metodo della teoria del diritto come pratica sociale è risolutivo: dalle azioni e dalle relazioni giuridiche nel loro insieme e nella loro migliore espressione si inferiscono le condizioni strutturali ed esplicative della loro realizzazione.

Se applichiamo questo procedimento alla pratica giuridica, incontriamo la difesa di diritti, l'imposizione di doveri, l'applicazione di sanzioni, la tutela di aspettative legittime, la risoluzione di conflitti mediante reintegrazioni,

24 Cfr. Postema, G.J., «Implicit Law», Law and Philosophy, 13 (1994), pp. 361-387. 
restituzioni, compensazioni e tutto questo, e altro ancora, alla luce di norme pubbliche dirette a garantire un ordine sociale stabile. Ma qui intendo focalizzare solo un aspetto della pratica giuridica, quello che è più direttamente collegato con il problema della coercizione. Tra gli altri suoi fini il diritto è rivolto a governare quei fatti sociali che sono manifestazioni di esercizio del potere dell'uomo sull'uomo e, quindi, fonte possibile di arbitrio.

Come si sa, il potere può essere arbitrario non solo per il suo titolo, ma anche per il suo esercizio. Il diritto intende contrastare l'arbitrarietà del potere in tutte le sue possibili forme, ma soprattutto quelle che trattano i destinatari delle regole come esseri non razionali, non liberi e non responsabili. Si tratta di un esercizio del potere che è arbitrario dal punto di vista antropologico, in quanto non rispettoso dei soggetti a cui si rivolge. Il diritto intende custodire nella misura del possibile l'agency nella vita sociale, cioè la capacità di deliberazione e di scelta, che è propria della dignità della persona umana. Questo è un obiettivo non facile da raggiungere, specie tenuto conto della necessità di limitare l'ambito di libertà delle azioni individuali a causa della coordinazione delle azioni sociali. Tuttavia, pur con queste restrizioni, le regole giuridiche si pongono come guida delle azioni e non già come loro causa.

Il modello causale del comando, per cui l'intenzione come stato mentale del comandante è volta a produrre l'obbedienza, cioè l'intenzione di seguire la regola come stato mentale del comandato, è fuorviante. Se la regola esercita un'azione causale, non si può dire che guidi un'azione, ma che tende a determinarla ed allora la coercizione diviene l'elemento centrale della regola stess $^{25}$. Significherebbe considerare il diritto come un mero meccanismo causale che tende a produrre, anche se non sempre con successo, l'azione voluta mediante minacce di conseguenze sfavorevoli, come un gigantesco processo di condizionamenti psico-fisici del comportamento di soggetti che si illudono di essere veri e propri agenti, ma in realtà non intenzionano il fine né scelgono i mezzi. Se il diritto dovesse essere così, come spesso di fatto è praticato, allora non sarebbe una pratica sociale per il suo concetto, ma una mera tecnica per il controllo sociale.

Il diritto rivela meglio la sua natura se guardiamo al comportamento dei destinatari delle regole giuridiche piuttosto che a quello delle autorità, cioè se

25 Per la critica del positivismo causale rinvio per tutti a CoYle, S.; PAVlakos, G. (eds.), furisprudence or Legal Science?, Hart Publishing, Oxford and Portland, 2005 e PaVlakos, G., Our Knowledge of the Law, Hart Publishing, Oxford and Portland, 2007. 
guardiamo all'applicazione e all'uso delle regole giuridiche più che alla loro produzione $^{26}$. Il diritto come pratica sociale privilegia il punto di vista dei destinatari rispetto a quello dei produttori ufficiali delle regole. Infatti, se l'azione di questi ultimi è controllata dal diritto, lo è al fine di tutelare i primi come i principali partecipanti della pratica giuridica. C'è, dunque, bisogno di una teoria giuridica ex parte populi, mentre abbondano le teorie del diritto ex parte principis.

Una teoria del diritto dal punto di vista dei consociati mette in primo piano il ruolo di «guida» che le regole giuridiche espletano nei confronti del loro comportamento. In questo ruolo di guida si manifesta il modo giuridico di limitare e controllare il potere, contrastando l'arbitrio. «Guidare un'azione» non si riferisce soltanto al modo in cui un'autorità dirige le azioni di coloro che governa, ma anche al modo in cui questi governano se stessi. Anzi, questo secondo senso è prioritario, in quanto è per permettere che agenti liberi e responsabili possano conservare ed esercitare le loro prerogative morali che l'autorità deve governare mediante il diritto. Il senso primario di guidare un'azione è quello che ognuno fa nei confronti di se stesso ${ }^{27}$. Questo è l'aspetto interno della regola di cui ha parlato Hart.

\section{LE RISORSE INTERNE DELLA PRATICA GIURIDICA}

Per verificare se una regola giuridica sia in grado di assolvere il ruolo di guida normativa del comportamento dei consociati, occorre guardare alle sue condizioni di praticabilità e queste si evidenziano compiutamente nella fase della sua applicazione, e non solo in quella della sua produzione. Ciò è mostrato da molteplici indizi ${ }^{28}$. Il concetto di diritto ha un carattere indiziario.

Un elemento centrale della pratica giuridica nelle sue forme più evolute è costituito da un sistema giuridico composto da norme prodotte da un'autorità. Esse devono essere ben identificabili al fine di poter essere seguite con consapevolezza dai destinatari e, pertanto, devono essere pubbliche. Queste norme devono anche avere un carattere generale. Non si tratta semplicemente

26 Cfr. VIOLA, F., «Tre forme di positività del diritto», in ZACCARIA, G., Diritto positivo e positività del diritto, Giappichelli, Torino, 1991, pp. 301-328.

27 Cfr. Viola, F., Rule of Law. Il governo della legge ieri ed oggi, Giappichelli, Torino, 2011, p. 47.

28 Per la silloge che segue ho tratto ispirazione dall'illuminante scritto di WALDRON, J., «The Concept and the Rule of Law», Georgia Law Review, 43 (2008), 1, pp. 1-61. 
di un'impossibilità pratica di governare le singole azioni dei destinatari delle norme mediante decreti ad hoc o comandi particolari, come pensava Austin ${ }^{29}$, ma ben più significativamente di lasciare ad essi uno spazio deliberativo, che può riguardare il se, il come e il quando dell'azione, cosa che sarebbe impedita da leggi ad personam. I princìpi dell'impersonalità e dell'eguaglianza sono alla base della generalità delle norme giuridiche. Ma anche le conseguenze della generalità sono rilevanti. Se le norme giuridiche sono generali, allora saranno anche necessariamente indeterminate. Il processo della loro determinazione si configura come un'attività cooperativa a cui concorrono non solo gli organi pubblici, ma anche i destinatari delle norme nella scelta delle loro azioni concrete. Questi ultimi, sia che intendano seguirle o violarle, applicano le norme (self-application), ne tengono conto nei loro processi deliberativi, le interpretano ed adattano ad esse le loro azioni ${ }^{30}$. Sono consapevoli che le loro interpretazioni possono essere controverse ed accettano di sottoporle ad uno scrutinio pubblico, nella convinzione di partecipare ad un'impresa comune in cui nessuno può rivendicare per sé un trattamento particolare se non per giustificati motivi.

Inoltre, in ogni pratica giuridica evoluta vi sono organi di giudizio deputati all'applicazione ufficiale delle norme nei casi controversi. Sembra anzi che queste istituzioni siano ancor più necessarie di quelle che producono le nor$\mathrm{me}^{31}$. Il loro modo di operare richiede tra l'altro che i giudici siano imparziali nei confronti delle parti in causa e indipendenti nei confronti di ogni potere, che vi sia il diritto di difesa e il contraddittorio tra le parti. Ulteriormente queste condizioni procedurali sono state perfezionate e raccolte nel principio costituzionale del «giusto processo», in cui tra l'altro l'opera del giudice è resa anche ancor più trasparente e la partecipazione delle parti ancor più incisiva ${ }^{32}$. Lo stesso obbligo di motivare le decisioni giudiziarie è da intendersi come

29 Austin, J., The Province of Furisprudence Determined (1832), ed. by W.E. Rumble, Cambridge University Press, Cambridge 1995, pp. 28-29.

30 Cfr. Waldron, J., «Self-application», NYU School of Law, Public Law Research, Paper N. 16-46, September 1, 2016. Non concordo però con Waldron quando nota che guidare un'azione è solo un modo di operare del diritto e non già un suo fine (p. 17), come invece sostiene - a mio avviso più correttamente - PERRY, S., «Hart on Social Rules and the Foundations of Law: Liberating the Internal Point of View», Fordham Law Review, 75 (2006), 3, pp. 1171-1209.

31 Cfr. Raz, J., Practical Reason and Norms (1975), Princeton University Press, Princeton, 1990, p. 132.

32 Per uno sviluppo in senso deliberativo del giusto processo con riferimento all'Italia cfr. Viola, F., «Una teoria deliberativa della giurisdizione?», Ars Interpretandi, 7 (2018), 1, pp. 13-28. 
un render ragione del giudizio assunto non solo nei confronti delle parti, ma anche di tutta la società, altrimenti non avrebbe senso la rilevanza della giurisprudenza e dei precedenti. Tutti gli aspetti di questa procedura non possono essere spiegati se si assume una visione meccanicistica o causale del diritto, che è volta a ridurre, se non ad eliminare, tutti i filtri che s'interpongono tra la volontà del legislatore e la condotta dei destinatari delle norme. Al contrario, la tendenza della civiltà giuridica è quella di accrescere le garanzie a tutela delle capacità deliberative non solo degli organi giudicanti, ma anche dei destinatari delle norme, affinché la legge sia una guida (e non una causa) delle azioni sociali. Si configura, pertanto, una forma di deliberazione collettiva, a cui partecipano titolari di ruoli differenti e il cui oggetto riguarda casi particolari da ricondurre nell'alveo della dimensione comune della vita associata. Il «caso» è per definizione un'istanziazione all'interno di una categoria generale che ne garantisce la socialità.

Nella pratica del diritto è presente anche una presupposizione che si può etichettare in modo approssimativo come il «principio della coerenza del sistema giuridico» ${ }^{33}$. Non si tratta in verità di una razionalità soltanto o puramente deduttiva, ma piuttosto di una ragionevolezza, spesso legata all'analogia e alla probabilità. Questo principio di carità intende affermare che il diritto non è mai il prodotto della sola volontà del potere, ma anche dall'opera della ragione. Questo principio ha un carattere chiaramente «normativo» e chiama in causa un altro gruppo di soggetti accanto ai legislatori e agli organi pubblici, cioè i giuristi. Che questo principio sia normativo, cioè che indichi come deve essere un sistema giuridico, è mostrato dalla constatazione che esso spesso di fatto non è né razionale né ragionevole. Di fatto un ordinamento giuridico si forma in modo alluvionale e per accumulazione. Una legge si aggiunge alle altre per stratificazioni successive durante un lungo periodo di tempo, che spesso contribuisce a cambiarne il senso. Certamente vi sono continui aggiustamenti mediante abrogazioni e modifiche legislative, che sono volte ad eliminare le contraddizioni e le antinomie più palesi. Ma a causa della pluralità delle interpretazioni legittime e della imprevedibilità dei casi concreti ve ne sono molte altre in agguato, pronte a presentarsi nel corso dell'applicazione del diritto. Il compito di armonizzare un sistema giuridico, di renderlo coerente e congruente e di conferire ad esso un'unitarietà di orientamento è proprio

33 Viene indicato come «sistematicy of law» da WALDRON, J., «The Concept and the Rule of Law», p. 32 e come «integrity of law» da DWORKIN, pp. 165-166. 
dei giuristi, che per la pratica del diritto sono partecipanti necessari quanto lo sono i funzionari e i giudici. Pertanto, il diritto come impresa cooperativa è opera della volontà e della ragione umana. Perché mai un sistema giuridico deve essere in certo qual modo coerente e unitario se non per svolgere il ruolo generale di guida delle azioni sociali?

Una legge può guidare o dirigere un'azione solo se è interiorizzata, cioè è assunta come criterio personale di azione. Ciò avviene mediante i processi di interpretazione e di argomentazione, che sono, insieme agli atti produttivi, i modi tipici di operare del diritto. L'obbedienza al diritto è essa stessa una pratica interpretativa e argomentativa. L'interpretazione vincola a qualcosa che i destinatari della regola devono rispettare, mentre l'argomentazione li rende partecipi attivi degli sviluppi della regola e della sua applicazione ai casi concreti. Proprio queste attività interpretative e argomentative mettono in luce anche che l'interiorizzazione della legge non significa che essa perda la sua dimensione sociale, ovvero che l'obbedienza diventi un fatto privato. In realtà, poiché v'è disaccordo sull'interpretazione e sul peso degli argomenti contrapposti, è necessario che queste attività siano pubbliche, siano soggette a scrutini pubblici e si concludano con una decisione autoritativa finale, com'è necessario nelle questioni pratiche. Una pratica giuridica è il luogo dei disaccordi ed è al contempo l'impresa di governarli ${ }^{34}$. Di conseguenza una pratica giuridica s'interroga continuamente su se stessa e sul modo migliore di realizzarsi, cioè sulla sua eccellenza, come è proprio di ogni pratica sociale secondo MacIntyre.

Per completare questo schizzo dei caratteri distintivi di una pratica giuridica, elaborato a partire dal punto di vista del contrasto all'uso arbitrario del potere, non resta che interrogarsi sulla sua virtù propria, ovvero sui suoi standards di eccellenza nei quali essa raggiunge i suoi fini. Certamente è necessario il rispetto delle procedure e delle forme giuridiche, perché esse garantiscono sia l'eguaglianza delle persone, sia il tessuto di comunanza delle azioni individuali. $\mathrm{Ma}$ - come abbiamo detto - con ciò stesso i disaccordi non sono eliminati, anzi al contrario spesso enfatizzati. Di fronte alla pluralità delle interpretazioni e delle argomentazioni legittime è necessaria una scelta, cioè una deliberazione guidata dalla ricerca del miglior diritto possibile nelle condizio-

34 Secondo Dworkin non solo v'è disaccordo sul modo d'interpretare le leggi, ma anche sullo stesso concetto di diritto, cioè sul modo d'intendere la stessa pratica giuridica, e non si può che dargli ragione. Cfr. Ivi, pp. 3-43. 
ni date. Migliore rispetto a che cosa? Le risposte possono essere molteplici: rispetto al bene della società intera, che tradizionalmente viene chiamato bene comune; rispetto all'applicazione migliore dei princìpi costituzionali, in cui la società riconosce i suoi valori fondamentali; rispetto a criteri di giustizia o a quelli che si ritengono tali. In ogni caso una pratica giuridica richiede attività di carattere deliberativo e, quindi, chiama in causa la distinzione tra scelte buone e scelte cattive. Non si può sostenere che in questo caso il bene è la conformità alla regola e il male la difformità, perché prima bisogna scegliere quale interpretazione corretta della regola seguire e quale argomento è più ragionevole. Certamente bisogna almeno riconoscere che è presente nella pratica giuridica una promessa di giustizia, spesso non mantenuta ${ }^{35}$. Un diritto palesemente ingiusto, per quanto valido possa restare, è sicuramente un insuccesso della pratica giuridica.

L'attuazione pubblica della giustizia è un valore comunitario ${ }^{36}$. Questo significa che è un valore non solo politico. $\mathrm{Ma}$ - come sappiamo - v'è ampio disaccordo su ciò che è giusto. Il diritto è volto a neutralizzare il più possibile gli effetti negativi di questo disaccordo, cercando di eliminare ciò che è sicuramente ingiusto: è ingiusto trattare le situazioni eguali in modo diseguale e quelle diseguali in modo eguale; è ingiusto trattare le persone in modo arbitrario, cioè come schiavi e non già come esseri che hanno diritto alla giustificazione degli atti di autorità al fine di guidare i loro comportamenti in modo consapevole e responsabile ${ }^{37}$; è ingiusto non rispettare la correttezza interpretativa e argomentativa delle pratiche giuridiche, che sono dettate da criteri formali ma non per questo avalutativi.

Poiché le istituzioni, le procedure e le forme giuridiche sono governate da valori interni ad esse, il loro esercizio richiede standards di eccellenza, che sono le virtù proprie dei partecipanti a quest'impresa cooperativa. La legge non può diventare diritto senza gli uomini e la loro abilità virtuosa. La legalità è anche una virtù, che consiste nella capacità di rispettare le regole traendo da esse il massimo di giustizia possibile in circostanze date ${ }^{38}$. Di conseguenza,

35 Cfr. Waldron, J., «Does Law Promise Justice?», Georgia State University Law Review, 17 (2000-2001), pp. 759-788.

36 RaWls, J., A Theory of Justice, revised edition, Oxford University Press, Oxford, 1999, p. 463.

37 Il diritto alla giustificazione come diritto fondamentale è affermato da FoRST, R., The Right to Fustification, Columbia University Press, New York, 2007.

38 Per questo rinvio a ViOLA, F., «La legalità come procedura e come prassi», Per la Filosofia, 10 (1993), n. 27, pp. 30-43. 
una pratica giuridica comporta sia modelli di eccellenza e di obbedienza alle regole, sia il conseguimento del valore interno della legalità ${ }^{39}$. Tuttavia, anche ammettendo che questi traguardi siano raggiunti dalla pratica giuridica, non per questo il diritto potrà essere giusto senza la giustizia politica. Da questo punto di vista il diritto positivo non è autosufficiente e, tuttavia, contribuisce a suo modo all'impresa generale della ricerca della giustizia nella vita associata.

Generalità e pubblicità delle leggi, principio del contraddittorio, coerenza del sistema giuridico, il ragionamento giuridico come controllo e sviluppo della prassi del diritto, la legalità come virtù volta a trarre dalla legge la risposta più giusta possibile, sono tutte caratteristiche strutturali della pratica giuridica dirette a salvaguardare il ruolo di guida della legge e, quindi, a difendere dall'uso arbitrario del potere. È facile notare che esse sono riassunte nella formula tradizionale del rule of law, che pertanto considero come la definizione del concetto stesso di diritto in quanto pratica sociale, e non già come una proprietà virtuosa di un sistema giuridico o una sua aspirazione ideale ${ }^{40}$.

Il rule of law, in questo contesto, non deve essere inteso come un insieme di princìpi presupposti dal diritto positivo ${ }^{41} \mathrm{o}$ di desiderata a cui esso deve risponde$\mathrm{re}^{42}$, ma più adeguatamente come un modo di strutturarlo e di praticarlo. Non si tratta solo di presupporre le condizioni di chiarezza normativa e di praticabilità del diritto, e neppure solo di sottolineare la necessità di istituzioni imparziali che le fanno valere, ma anche di mostrare come queste pratiche si configurino come una forma di vita che struttura le nostre azioni ${ }^{43}$. La legittimità del diritto riposa su queste pratiche immanenti nel diritto stesso $^{44}$. Queste a loro volta, in quanto si rivolgono agli aspetti più importanti e rilevanti della vita associata, si estendono a tutta la comunità politica e si presentano come stabili. La stessa stabilità sociale è una difesa dall'arbitrio del potere. Di conseguenza, il diritto

39 MACINTYRE, p. 190.

40 Cfr. Viola, F., «Il Rule of Law e il concetto di diritto», Ragion pratica, 30 (2008), pp. 151-168.

41 Arthur Kaufmann li esemplifica nel principio di eguaglianza, in quello di buona fede, nell'imperativo pacta sunt servanda, nel principio di colpevolezza, in quello del bilanciamento di diritti e doveri, nella regola aurea. Cfr. Kaufmann, A., Analogie und «Natur der Sache». Zugleich ein Beitrag zur Lebre vom Typus (1965), Decker \& C.F. Müller, Heidelberg, 1982, Kapitel II.

42 Cfr. Fuller, L.L., The Morality of Law, revised edition, Yale University Press, New Haven and London, 1969, chap. II. Tuttavia le tesi di Fuller sono pienamente compatibili con la visione del diritto come pratica sociale qui difesa.

43 Cfr. Simmonds, N.E., «Law as an Idea We Live By», in Duke, G.; George, R.P. (eds.), The Cambridge Companion to Natural Law Jurisprudence, Cambridge University Press, New York, 2017 , p. 245.

44 Cfr. Dyzenhaus, D., «Law and Public Reason», McGill Law fournal, 38 (1993), pp. 366-393. 
positivo, pur essendo artificiale, è fondato su ragioni morali ${ }^{45} \mathrm{e}$ richiede come sua giustificazione giudizi morali ${ }^{46}$. Questi giudizi hanno un carattere etico-politico ambivalente, in quanto sono rivolti sia contro l'anarchia sia contro l'uso arbitrario del potere. Per questo il diritto si occupa di tutti i campi in cui è possibile esercitare la violenza o in qualche modo arrecare offesa ad altri (iniura), sia nei rapporti privati sia in quelli pubblici.

In conclusione, una teoria del diritto come pratica sociale non può assumere la prospettiva neutrale della terza persona, cioè quella puramente descrittiva dei fatti sociali e degli enunciati normativi, perché si pone dal punto di vista dei partecipanti alla pratica, pur se a vario titolo e con diversi ruoli. Non può neppure assumere la prospettiva della prima persona, che è propria della scelta morale, perché qui non sono in primo piano le intenzioni soggettive dei partecipanti alla pratica giuridica, ma quelle che essi dovrebbero avere per il fatto di prender parte ad un'attività cooperativa. Il punto di vista privilegiato della teoria del diritto come pratica sociale è quello della seconda persona, cioè dell'alterità, come conviene alle questioni di giustizia (iustitia est ad alterum), in cui sono in gioco i diritti e i doveri dei partners delle relazioni sociali significative. In quest'ottica intersoggettiva le ragioni per agire sono quelle che anche gli altri possono accettare e che sono in grado di consolidarsi come ragioni comuni o ragioni pubbliche a sostegno della vita sociale, sia a livello delle istituzioni fondamentali sia sul piano dei contenuti delle regole giuridiche. $\grave{E}$ ovvio che questo è un cantiere sempre aperto con continui aggiustamenti, incessanti revisioni e questioni controverse non risolte una volta per tutte. All'interno della pratica giuridica esiste un diritto fondamentale dei partecipanti all'eguale giustificazione delle regole, affinché la loro imposizione non sia frutto di arbitrio o di manifesta irragionevolezza, né si traduca in oppressione, discriminazione e dominio ${ }^{47}$. A queste procedure di giustificazione tutti dovrebbero partecipare come individui liberi ed eguali ${ }^{48}$.

$45 \ll$ A theory of law is, unavoidably, a reflection upon our form of moral association». SIMMONDS, p. 250.

$46 \ll$ Moral premises are required in sound reasoning to the conclusion that a community does or does not (more or less) attain the rule of law». ENDICOTT, T., «The Reason of the Law», The American Fournal of Jurisprudence, 48 (2003), p. 83.

47 Cfr. Forst, R., Fustification and Critique. Towards a Critical Theory of Politics, Polity, Cambridge, 2013.

48 Forst, R., «Two Pictures of Justice», in ID. (ed.), Fustice, Democracy and the Right to fustification. Rainer Forst in Dialogue, Bloomsbury, London, 2014, pp. 3-26. Cfr. anche più di recente GIOVANOLA, B., Giustizia sociale. Eguaglianza e rispetto nelle società diseguali, Il Mulino, Bologna, 2018. 


\section{LA COERCIZIONE DEL DIRITTO}

$\grave{E}$ ora venuto il momento d'interrogarci sul modo in cui la coercizione deve essere considerata alla luce del diritto come pratica sociale, cioè se e in che senso essa appartiene alla natura del diritto così inteso.

S'è già detto che la coercizione proveniente dall'esercizio del potere in tutte le sue manifestazioni è già presente abbondantemente nella vita sociale e politica, e che uno degli obiettivi del diritto è senza dubbio quella di regolamentare l'uso della forza pubblica per custodire nella misura del possibile l'esercizio dell'agency. Tuttavia è sotto gli occhi di tutti che si genera una coercizione che ha la sua origine e la sua fonte nel diritto stesso. Non mi riferisco alle sanzioni non coattive, che si trovano in tutte le pratiche sociali come reazioni critiche nei confronti dei comportamenti devianti, ma piuttosto alla coercizione in senso stretto, che è quella decisiva. Le stesse sanzioni giuridiche non coattive alla fin dei conti poggiano sull'uso della forza come risorsa ultima nel caso della loro inefficacia. Né vale affermare che per il diritto è necessaria solo la minaccia dell'uso della forza e non già la sua attuazione ${ }^{49}$, perché la deterrenza è efficace solo quando è prevedibile l'attuazione delle minacce. Insomma, c'è un uso della forza propriamente giuridico che sembra essere costitutivo della stessa natura del diritto, ma questo si pone in contraddizione con quel fine del diritto che consiste nella custodia delle capacità deliberative delle persone in quanto aspetto fondamentale della tutela della loro dignità. Quest'uso della giustizia violenta, minacciato e attuato da parte del diritto, è quello che Ricoeur ha icasticamente bollato come «lo scandalo della pena» ${ }^{50}$.

Né qui vale richiamare il noto brocardo coactus voluit, sed voluit, perché non si tratta di stabilire se v'è stata o meno la volontà dell'azione ${ }^{51}$, ma se questa sia il risultato di un atto di dominio dell'uomo sull'uomo. A questo proposito è improprio richiamare l'esempio aristotelico dei naviganti che gettano in mare il carico per salvare la vita ${ }^{52}$, perché la tempesta è una circostanza che

49 Cfr. Green, L., «The Force of Law: Duty, Coercion, and Power», Ratio furis, 29 (2016), 2, p. 167.

50 Ricoeur, P., Le Droit de punir, Intervention à la commission Nationale Justice et Aumônerie des Prisons sur «Le Sens de la peine», Paris, 2001.

51 Secondo Aristotele la particolarità dell'azione è il luogo per giudicare della sua volontarietà o involontarietà. Le azioni compiute per costrizione sono involontarie nella causa, ma volontarie nella scelta.

52 Etica Nicomachea, L. III, 1, 1110a 10. 
non dipende da una volontà umana; e neppure è appropriato il noto esempio del bandito che costringe a consegnare la borsa per salvare la vita, perché nella sanzione giuridica il dominio dell'uomo sull'uomo è proveniente dal diritto stesso, il cui fine - come s'è qui finora sostenuto - è proprio quello di contrastarlo. Se il diritto operasse come il bandito, sarebbe null'altro che una forma di esercizio del potere tra le altre.

Se il fine del diritto è quello di combattere l'arbitrio del potere e di difendere l'autonomia morale delle persone nelle loro scelte di vita, il suo ricorso alla coercizione in senso stretto si presenta, dunque, come contraddittorio. Il paradosso che bisogna spiegare è, dunque, quello di un rimedio che ha la stessa natura del male che intende esorcizzare. Non è neppure convincente affermare che la coercizione di per sé non sarebbe propria del diritto se la pratica giuridica non fosse interconnessa con quella politica ed economica, perché è proprio questa interconnessione che la definisce a dispetto di tutti i tentativi di isolare il diritto.

Una prima considerazione riguarda la presenza diffusa di istituzioni create dal diritto che direttamente applicano ed esercitano la coercizione (enforcement institutions), come emblematicamente sono quelle di polizia o quelle carcerarie. A ben guardare, però, la presenza di queste istituzioni è propria del diritto statale, che è solo una specie del genere «diritto». Per la concezione statalista del diritto la sanzione è interna, in quanto è irrogata dagli organi stessi del sistema che l'ha stabilita, e fisicamente costrittiva ${ }^{53}$. Com'è ben noto, lo Stato di diritto intende sottoporre tutte le sue manifestazioni al controllo giuridico e, conseguentemente, il diritto stesso assume tendenzialmente come proprie tutte le prerogative dello Stato. Ma, se ad esempio guardiamo al diritto internazionale contemporaneo, possiamo constatare che spesso manca il meccanismo della coercizione fisica, minacciata o esercitata. Quando è necessario ricorrere ad essa, si affida il compito ad organi esterni al sistema giuridico internazionale, che spesso sono quelli propri degli Stati (externalized enforcement). Ciò non vuol dire che manchino forme di reazione alla violazione degli obblighi internazionali. Queste spesso consistono nella minaccia dell'esclusione (outcasting), cioè della privazione dei benefici della

53 Questo è il vero e proprio significato della concezione weberiana, cioè la gestione diretta da parte dello Stato anche dell'applicazione delle sanzioni coattive. Infatti il monopolio dell'autorizzazione ad usare la forza pubblica è già presente nel pensiero politico di Aristotele. Cfr. BRADY, P., «Coercion, Political Authority and the Common Good», The American fournal of furisprudence, 62 (2017), 1, pp. 75-87. 
cooperazione sociale o economica e dell'appartenenza, cioè in sanzioni non coattive $^{54}$. Tuttavia non possiamo certamente sostenere che non sia propria della natura del diritto l'istituzione giudiziaria, che, pur non eseguendo l'atto coercitivo, lo dispone e autorizza.

Anche se le istituzioni giuridiche direttamente deputate all'uso della forza possono mancare, non per questo il diritto perde il suo carattere coercitivo, poiché a questo fine basta autorizzare l'uso della forza e prescrivere l'irrogazione di sanzioni nel caso di violazione delle direttive giuridiche, anche se poi la forza viene di fatto esercitata da altri all'esterno del sistema giuridico (come nel caso del cd. «braccio secolare»). Anche nel caso di ricorso ad organi esterni che comminano la sanzione, c'è pur sempre bisogno dell'autorizzazione ad irrogarla. Nella sostanza c'è ben poca differenza tra punire direttamente ed autorizzare altri a punire. Per questa via non si risolve certamente il problema del rapporto tra l'uso della forza e l'esercizio dell'agency.

L'argomento più diffuso per giustificare la natura coercitiva del diritto è quello della sua necessaria efficacia ${ }^{55}$. Una regola giuridica inefficace, per quanto resti formalmente valida, ha fallito il suo obiettivo che è quello di dare stabilità alle relazioni sociali. Se poi è largamente inefficace nel suo complesso tutto il sistema giuridico, allora è fallita tutta l'impresa giuridica. In quest'ottica la sanzione è giustificata in quanto è volta ad assicurare l'efficacia del diritto. Tuttavia questo argomento ha poca presa nella teoria del diritto come pratica sociale, poiché essa per definizione assume che il diritto sia già in qualche modo efficace, cioè che già esista una pratica giuridica diffusa e consolidata. In quest'ottica il carattere coercitivo del diritto ha un ruolo subalterno o collaterale, in quanto costituisce l'ultima risorsa a cui far ricorso quando tutte le altre ragioni per obbedire al diritto sono inefficaci.

La convinzione della pervasività della coazione giuridica è dovuta in buona parte alla sua maggiore visibilità rispetto agli atti di conformità alle regole giuridiche e alle ragioni che li sostengono. La normalità è meno visibile degli atti volti alla sua trasgressione, ma è ben più frequente. Una pratica giuridica

54 Questo tipo di sanzioni sono analizzate accuratamente da HathaWAY, O.A.; SHaPIRO, S.J., «Outcasting: Enforcement in Domestic and International Law», The Yale Law fournal, 121 (2011), pp. 252-349.

55 E stato anche sostenuto che non rientra nel concetto di diritto la sua efficacia. Ma è ben più accettabile la tesi per cui un sistema giuridico aspira necessariamente ad essere efficace. Cfr. FISSEL, B.M., «Sanctions and Efficacy in Analytic Jurisprudence», Rutgers University Law Review, 69 (2017), pp. 1627-1652. 
poggia sul grado di socializzazione, sui benefici che provengono da un atteggiamento cooperativo, dalla moralità positiva e, non da ultimo, dal sostegno che proviene da altre pratiche sociali, come quelle religiose e del costume sociale $^{56}$. Si può senza dubbio sostenere con ragione che anche tutte queste sono forme di pressione sociale che inducono ad atti di conformità nei confronti del sistema giuridico. Ma non si tratta a rigore di vera e propria coercizione, qual è quella proveniente dal potere, quanto piuttosto di formazioni consuetudinarie necessariamente prodotte dalla vita comune. In ogni caso l'argomento dell'efficacia dimostra soltanto che un sistema giuridico deve essere nel suo complesso sostenuto da un'adeguata pressione sociale e, se del caso, rafforzato da qualche forma di reazione critica, ma non già che questa coercizione debba essere necessariamente giuridica, cioè che il diritto in quanto tale sia internamente coercitivo ${ }^{57}$. Da questo punto di vista è possibile concepire un diritto pienamente efficace sprovvisto di sanzioni propriamente giuridiche.

\section{LA COERCIZIONE GIURIDICA IN GENERALE}

La teoria del diritto come pratica sociale rigetta sia la concezione di $\mathrm{Au}-$ stin per cui la coercizione è il genere e la legge giuridica una sua specie, sia quella di Kelsen per cui è la coercizione a rendere il diritto una forma distinta di ordine sociale, sia quelle di Bentham e Holmes per cui la coercizione è l'elemento più saliente e significativo del diritto. Per il diritto come pratica sociale il carattere coercitivo del diritto non è costitutivo ma derivato, non è strutturale ma conseguenziale. Esso è giustificato dalla pretesa del diritto di avere autorità nella regolamentazione della vita sociale e dall'accettazione di essa da parte dei suoi destinatari. Questi riconoscono che i diritti e i doveri giuridici hanno una rilevanza particolare nella deliberazione pratica, perché conferiscono stabilità e continuità alla vita sociale, difendono dall'arbitrio del potere e promettono il perseguimento della giustizia. La presenza delle sanzioni giuridiche è giustificata normativamente da queste finalità strutturali del diritto.

Nell'ottica di una pratica sociale il diritto è riconosciuto come tale non per il fatto di essere coercitivo, ma nella misura in cui offre una struttura

56 Il ricorso alla coercizione aumenta nella misura in cui diventa più debole la coesione sociale, com'è proprio delle società contemporanee segnate dal pluralismo.

57 Lamond, G., «Coercion and the Nature of Law», Legal Theory, 7 (2001), pp. 47-50. 
stabile di regole che rende possibile una vita sociale tendenzialmente libera dal dominio dell'uomo sull'uomo ${ }^{58}$. Vedere nella coercizione l'elemento essenziale, o tipico del diritto, quell'elemento che demarca o differenzia il diritto dagli altri ambiti della vita pratica, significa scambiare l'effetto per la causa. Il diritto è coercitivo come conseguenza delle ragioni per cui è bene che vi sia. Queste ragioni sono la giustificazione della coercitività del diritto e la causa del suo essere coercitivo, che ne è una loro conseguenza non necessaria ${ }^{59}$.

Da questa giustificazione normativa delle sanzioni si possono trarre ragioni per agire in conformità ai precetti giuridici, ragioni di supporto o di rafforzamento, ulteriori rispetto a quelle tratte dal contenuto dei precetti. Queste ragioni, che bussano alla porta della deliberazione pratica, possono essere così individuate: la sanzione, per la sua natura, forma e configurazione giuridica, indica il grado d'importanza che il precetto riveste per la stabilità della vita sociale, ed è per questo che deve essere proporzionata al grado del valore che s'intende difendere ${ }^{60}$; inoltre, la sanzione si presenta anche come un atto di giustizia correttiva, che intende contrastare gli ingiusti vantaggi ottenuti da chi ha disobbedito alla legge nei confronti di coloro che invece l'hanno obbedita, ripristinando in certo qual modo l'eguaglianza ${ }^{61}$; infine, la sanzione è la logica conseguenza della pretesa di giustizia che ogni sistema giuridico avanza, anche quello più ingiusto, poiché ci si aspetta che soprattutto la violazione delle leggi giuste sia punita ${ }^{62}$. Sia la funzione simbolica della sanzione, sia la funzione di assicurazione che genera fiducia nel sistema giuridico, sia la funzione di rafforzamento della pretesa di giustizia sono tutte ragioni pienamente compatibili con il rispetto dei processi deliberativi, anche se nessuna di esse è decisiva e ognuna di esse è suscettibile di un uso ideologico.

58 VINX, L., «Schauer on the Differentiation of Law», in BEZEMEK, C.; LADAVAC, N. (eds.), The Force of Law Reaffirmed. Frederick Schauer Meets the Critics, Springer, Switzerland, 2016, p. 132.

59 È interessante notare che Tommaso d'Aquino, pur sostenendo che la vis coactiva è una proprietà necessaria della legge (Sum. Theol., I-II, 96, 5), non la include nel suo concetto generale di legge, tutto centrato sulla vis directiva (Sum. Theol., I-II, 90, 4). Cfr. VIOLA, F., «Aquinas (On Natural Law)», in M. Sellers e S. Kirste (eds.), Encyclopedia of the Philosophy of Law and Social Philosophy, Springer, Dordrecht, 2019.

60 Cfr. D’Agostino, F., La sanzione nell'esperienza giuridica, V ed., Giappichelli, Torino, 1999, pp. 3-62.

61 Cfr. Finnis, J., Natural Law \& Natural Rights, II ed., Oxford University Press, Oxford, 2011, pp. 260-264.

62 Cfr. Lagerspetz, E., «Obligations, Sanctions, and Coercion», in Chiassoni, P. (ed), The Legal Ought, Giappichelli, Torino, 2001, p. 139. 
Nonostante la presenza di queste giustificazioni normative della sanzione giuridica $^{63}$, resta il fatto che nell'opinione comune la prescrizione di sanzioni viene primariamente intesa come una forma di deterrenza, che con la minaccia di svantaggi e di pene è diretta a costringere i destinatari all'obbedienza dei precetti giuridici a prescindere dalla considerazione del loro contenuto. A questo punto sembrerebbe che la deliberazione dei destinatari del precetto sia gravemente compromessa se non del tutto paralizzata. Il timore della pena, infatti, non si presenta propriamente come una ragione per obbedire al precetto, ma come un motivo che ostacola la libertà della deliberazione. Quando si dice che la legge costringe a fare ciò che non si vuole fare, ci si riferisce propriamente e precisamente a questo ruolo della sanzione giuridica, particolarmente incisivo in quella coattiva.

\section{LEGGE GIURIDICA E COERCIZIONE}

Molti giuspositivisti sostengono che il concetto di legge giuridica in quanto tale debba essere indipendente dal suo contenuto, se la si vuole distinguere dalla legge morale. Di conseguenza, l'obbedienza alla legge è giustificata o dal rispetto dell'autorità o dal timore delle sanzioni.

Alla luce della ragion pratica, invece, l'obbedienza al diritto dovrebbe essere una decisione come risultato di una deliberazione consapevole e libera, fondata sul contenuto dei precetti giuridici. Sappiamo bene, però, che di fatto si obbedisce al diritto per motivi molto diversi fra loro: per abitudine, per acquiescenza, per paura delle sanzioni, per mero rispetto dell'autorità e, in ultimo, per convinzione della giustezza della regola. La pratica giuridica li considera tutti come motivi legittimi. Tuttavia, qualora prevalesse di gran lunga l'obbedienza per il solo timore delle sanzioni, non ci troveremmo più di fronte ad una pratica giuridica, ma ad un'ulteriore forma di violenza sociale mascherata.

L'obbedienza alla legge in quanto tale, cioè indipendentemente dal suo contenuto, è anch'essa fornita di una giustificazione morale, che è meno esile di come appare. Infatti essa riposa sul riconoscimento della legittimità dell'autorità. Sarebbe una contraddizione performativa accettare la legittimità

63 Per la critica del carattere eticamente neutrale della sanzione cfr. FowLER, M., «Coercion and Practical Reason», Social Theory and Practice, 8 (1982), 3, pp. 329-355. 
dell'autorità e non ciò che essa implica, cioè le leggi da questa emanate. A questa contraddizione solo un anarchico potrebbe sfuggire.

Il riconoscimento della legittimità dell'autorità è a sua volta fondato su più profonde ragioni. Si può pensare che un'autorità sia necessaria per il valore della coordinazione delle azioni sociali. Da questo punto di vista, anche se una legge è ritenuta ingiusta, inopportuna o non conveniente, la disobbedienza non produrrebbe una maggiore coordinazione sociale, anzi l'ostacolerebbe vieppiù. Questa è spesso la ragione che induce ad obbedire ad una legge che riteniamo ingiusta o molto imperfetta, cioè il valore dell'ordine sociale che essa in ogni caso difende. Un'altra ragione per obbedire alla legge in quanto tale riposa sulla fiducia nelle maggiori capacità epistemiche dell'autorità. Anche se non si concorda sul contenuto della legge, tuttavia si riconosce che il proprio punto di vista non è in grado di giudicare sulla base del bene comune, anche perché manca delle informazioni necessarie. Si sospende, pertanto, il proprio giudizio e si fa proprio quello dell'autorità. Mentre la precedente motivazione si basava sul potere, questa si fonda sul sapere di chi è più competente e ha per fine istituzionale il bene comune. Per questo prende in considerazione in qualche modo il contenuto della legge, anche se non vi aderisce sulla base del proprio giudizio, ma di un atto di affidamento, come spesso è necessario nelle relazioni sociali.

Anche l'obbedienza dettata dal timore di evitare le conseguenze spiacevoli della sanzione può essere fondata su ragioni in qualche modo morali. Ciò avviene quando la sanzione giuridica non è considerata come un puro atto di arbitrio da parte del più forte, ma come l'esercizio di un diritto logicamente legato al ruolo dell'autorità, al fine di indurre ad obbedire anche coloro che non condividono il contenuto delle leggi. Questo atteggiamento mentale è ben diverso da quello di chi soggiace ad un puro atto di forza, che lo costringe a fare ciò che non desidera e a cui non può resistere, come nel noto esempio del bandito. C'è grande differenza fra riconoscere la legittimità della coercizione e soggiacere ad essa per debolezza.

Infine, bisogna notare che obbedire ad una norma giuridica per ragioni morali legate al suo contenuto non significa affatto non considerare questa obbedienza come rivolta alla norma giuridica in quanto tale. Infatti nel concetto esemplare di norma giuridica è senza dubbio compresa la sua pretesa di giustizia, che riguarda il suo contenuto. Una norma ritenuta ingiusta dal suo destinatario non è ai suoi occhi ciò che una norma giuridica dovrebbe essere nel suo senso pieno, anche se ne riconosce la validità formale. Nel concetto pieno di norma giuridica in quanto tale è compresa la sua giustizia. 
L'atto di obbedienza (o di disobbedienza) alla legge in quanto atto umano è (o dovrebbe essere) normalmente preceduto da una deliberazione, in cui spesso sono presenti anche ragioni morali di vario genere, dirette ed indirette. Il diritto stesso crea o incorpora ragioni morali per obbedire ad esso, affinché seguire le sue regole sia un atto di scelta da parte di esseri razionali e liberi. Il rispetto dell'agency nella vita sociale è possibile solo se le norme giuridiche possono costituire parte integrante del ragionamento pratico in cui consiste il processo di deliberazione, e non già quando lo impediscono o lo escludono. Una teoria del diritto come pratica sociale non può esimersi dal conferire un'importanza centrale alle ragioni dell'obbedienza al diritto e ai suoi processi deliberativi. Se è vero che i sistemi giuridici sono istituzioni fornite di giustificazioni morali ${ }^{64}$, allora queste giustificazioni operano come ragioni nella pratica del diritto.

Tra le concezioni sul modo in cui operano le regole giuridiche, due in particolare non sono compatibili con la teoria del diritto come pratica sociale.

Com'è ben noto, secondo Raz i dettami dell'autorità sono uno speciale tipo di ragioni per agire, in quanto esse escludono per principio il processo deliberativo in cui si confrontano ragioni contrapposte di primo livello. Sono, dunque, exclusionary reasons. Ciò è necessario al fine di assicurare quella coordinazione delle azioni sociali che è il compito proprio dell'autorità ${ }^{65}$. Raz aggiunge che affidarsi al giudizio dell'autorità non significa agire irrazionalmente o arbitrariamente, perché vi sono solide ragioni per fondare la necessità di un'autorità. Pertanto questa concezione appartiene a quelle che considerano l'obbedienza alla legge giuridica in quanto tale come indipendente dal suo contenuto. Ma, se è vero che vi sono ragioni forti per obbedire all'autorità, non è vero che per ciò stesso venga bloccato il processo deliberativo. Basti solo notare che la pluralità delle interpretazioni della legge rimette in gioco ai fini della scelta le stesse ragioni di primo livello a supporto della decisione finale ${ }^{66}$. Per quanto il diritto sia un mondo artificiale, per essere operativo, cioè dal punto di vista della ragion pratica, non può fare a meno di ricorrere anche alle ragioni naturali nel momento della sua applicazione.

L'altra prospettiva, anch'essa non accettabile dal punto di vista della teoria del diritto come pratica sociale, è quella di Frederick Schauer. Per mostrare

64 HathaWay; Shapiro, p. 255.

65 RAZ, pp. 64-65.

66 Per una critica ben più articolata di queste tesi di Raz rinvio a RodrigueZ-BLANCO, V., Law and Authority Under the Guise of the Good, Hart Publishing, Oxford and Portland, 2014, pp. 139-160. 
quanto sia rilevante la pervasività della coercizione nel diritto, egli definisce la legge giuridica come quella direttiva che induce a fare cose che non desideriamo fare per molteplici ragioni, tra cui vi sono anche quelle morali ${ }^{67}$. Il concetto di coercizione giuridica non si dovrebbe ridurre, dunque, a quello dell'uso della forza nelle sue diverse e appropriate manifestazioni, ma già è insita nello stesso dettato precettivo che cancella la rilevanza dei nostri desideri, per quanto giustificati possano essere. Pertanto, la legge giuridica in quanto tale è già di per sé coercitiva e quella che si considera a stretto rigore come coercizione, cioè l'uso della forza, non è altro che la sua logica conseguenza. Infatti, qualora la legge giuridica fosse obbedita per altre ragioni diverse dalla sua forza impositiva, allora perderebbe il suo carattere distintivo rispetto alla legge morale.

Come si può facilmente notare, non siamo lontani dalla concezione di Raz, ma con una differenza significativa. L'ottica di Schauer non è normativa, ma descrittiva in senso sociologico e psicologico (e quindi la sua validità dipende da dati empirici, per altro discutibili). Si può dire che sia la trascrizione delle exclusionary reasons nei termini della psicologia dell'obbedienza alla legge giuridica. Quindi non c'è da stupirsi se anche qui abbiamo una paralisi del processo deliberativo, questa volta non sul piano normativo delle ragioni di ordine superiore, ma dell'efficacia della minaccia di conseguenze spiacevoli in seguito alla disobbedienza.

Tutto ciò non è convincente. L'affermazione che una regola giuridica è praticamente rilevante quando richiede di agire in contrasto a ciò che faremmo se non ci fosse questa legge, non implica necessariamente che questa abbia un carattere coercitivo, perché la sua presenza potrebbe portare nuove ragioni per agire che in sua assenza non sarebbero state prese in considerazione. Ma, se così fosse, cioè se obbedissimo alla legge per queste ragioni, allora secondo Schauer essa risulterebbe lo stesso giuridicamente irrilevante, perché in questi casi desidereremmo fare proprio ciò che la legge prescrive di fare. In tal modo si misconosce la differenza tra il carattere direttivo della legge giuridica e quello coattivo. Una regola è normativa quando è in grado di fornire ragioni morali per agire in un certo modo ${ }^{68}$. D'altronde non è detto che la forza direttiva della legge giuridica debba essere la stessa di quella della legge morale,

67 Schauer, F., The Force of Law, Harvard University Press, Cambridge, Mass., 2015, p. 1.

68 Bustamante, T., «Coercion and the Normativity of Law: Some Critical Remarks on Frederick Schauer's The Force of Law», in BEZEMEK; LADAVAC (eds.), p. 33. 
proprio perché si pone - come s'è già detto - nella prospettiva della seconda persona e del bene comune.

Va da sé che in generale le leggi esistono perché si potrebbe agire in senso contrario se non vi fossero. Esistono per guidare azioni contingenti di per sé aperte alle più diverse e opposte determinazioni. Persino nel caso del rispetto della legge giuridica in quanto tale, cioè indipendentemente dal suo contenuto, non c'è necessariamente l'abbandono della sua funzione direttiva. Si dovrebbe semmai dire che, mentre le leggi morali portano ragioni intrinseche per agire, quelle giuridiche presentano almeno ragioni estrinseche basate su valori sociali (la coordinazione delle azioni e delle libertà, l'ordine sociale, la sicurezza e così via). Queste ragioni estrinseche rispetto ai contenuti della legge sono ritenute talmente indispensabili per la vita associata da pretendere un ruolo significativo nel processo deliberativo, che riguarda l'obbedienza alla legge, e da giustificare come ultima risorsa l'uso della forza pubblica. Come sappiamo, il modello del bandito è fuorviante sotto due profili: sia perché questi non è stato autorizzato a minacciarci, sia perché il suo ordine non riposa su ragioni estrinseche socialmente rilevanti. Schauer, a differenza di Hart, non guarda agli aspetti sociali della regola, ma alle motivazioni psicologiche degli individui ${ }^{69}$, ma con ciò perde di vista la peculiarità di una pratica sociale.

\section{LA COERCIZIONE GIURIDICA IN SENSO STRETTO}

Nonostante tutte queste considerazioni, che valgono per le sanzioni in generale, l'uso della forza fisica non si armonizza con gli standard di eccellenza della pratica giuridica e resta per essa un problema da risolvere.

Il paradosso del diritto, che usa la forza per contrastare la forza, sembra superato dalla teoria che vede nella coazione non già lo strumento del diritto, ma il suo oggetto proprio da sottoporre alla regolamentazione giuridica ${ }^{70}$. La teoria del diritto come regola della forza a prima vista si presenta per il diritto come pratica sociale come attraente da molteplici punti di vista: mette a fuoco l'obiettivo del diritto di combattere l'arbitrio del potere; identifica il diritto

69 Ivi, p. 34.

70 Com'è ben noto, questa teoria è stata ripresa e ulteriormente sviluppata da Bobbio che ne ha messo in luce gli aspetti e i vantaggi principali. Cfr. BовBIO, N., «Diritto e forza», ora in ID., Studi per una teoria generale del diritto, a cura di T. Greco, Giappichelli, Torino, 2012, pp. 101118. 
non già con l'uso della forza ma con la sua regolamentazione; individua un contenuto materiale per la regola giuridica e, non da ultimo, considera il diritto come un ordinamento, cioè nella sua totalità o non già nelle sue singole parti, anche se si tratta pur sempre di un ordinamento di norme e non di azioni. Tuttavia a ben guardare queste convergenze sono soltanto apparenti e in realtà v'è una radicale incompatibilità fra le due prospettive.

Abbiamo detto che l'arbitrio del potere non si manifesta soltanto nell'uso della forza, ma anche nell'instabilità della società e nella frustrazione delle aspettative sociali. In quest'ottica l'idea che il diritto abbia come suoi diretti destinatari i funzionari pubblici e non già tutti i cittadini e che, conseguentemente, l'ordinamento giuridico si riduca nella sostanza alle norme secondarie, è in contrasto con l'idea stessa di una pratica sociale, che implica un'accettazione diffusa da parte di tutti i destinatari del diritto e non solo degli organi pubblici. La teoria del diritto come regola della forza è una teoria ex parte principis e non ex parte populi. Inoltre, bisogna anche interrogarsi su quali criteri seguire nell'organizzare l'uso della forza pubblica. Non si tratta soltanto di stabilire il chi, il come e il quanto, ma anche il quando, cioè quando l'uso della forza è legittimo ovvero a quali comportamenti esso debba essere collegato. $\mathrm{E}$ a questo punto non solo il ruolo delle norme primarie riassume tutta la sua importanza e centralità, ma anche l'esigenza della stabilità sociale fa sentire la sua voce. Se non fosse così, avremmo solo un arbitrio organizzato, che non per questo cesserebbe di essere un arbitrio, forse ancor più minaccioso di quello privo di regole.

Poiché il carattere violento della sanzione giuridica è - come s'è detto paralizzante per la deliberazione, la teoria del diritto come pratica sociale mira a ridurlo al minimo indispensabile. Come abbiamo detto, si tratta di una teoria normativa che guarda al dover essere del diritto e all'eccellenza della sua pratica. Poiché il dover essere appartiene all'essere del diritto, non è improprio considerarla come una teoria a tutti gli effetti. Ma la progressiva riduzione del ricorso alla forza fisica è anche un trend evolutivo ben presente nel processo, sempre incompiuto, di civilizzazione del diritto.

Proverò ora solo ad abbozzare il modo in cui la teoria del diritto come pratica sociale dovrebbe considerare le sanzioni giuridiche.

Se gettiamo uno sguardo molto generale ai diversi tipi di sanzione giuridica, che sono anche un modo diverso d'intenderla, possiamo distinguere le sanzioni privative e quelle costruttive. Sono privative (o costrittive) le sanzioni che hanno per fine solo quello di infliggere uno svantaggio nel caso di una violazione della regola giuridica. Sono costruttive quelle che mirano ad un 
effetto positivo, anche se per vie coercitive. È evidente che solo le sanzioni privative possono essere minacciate e, quindi, hanno una funzione di deterrenza, mentre la possibilità di applicare le sanzioni costruttive si verifica solo dopo la violazione della regola giuridica e a specifiche condizioni.

Le sanzioni privative sono di due tipi: le sanzioni di esclusione e le sanzioni punitive. Le prime non riguardano l'uso della forza fisica, ma - come s'è già detto - l'esclusione dall'attività cooperativa o in generale dalla coordinazione sociale in un determinato ambito di attività. Si tratta di forme di cooperazione sociale a cui si è aderito volontariamente, così come si gioca di propria volontà. Se non si rispettano le regole del gioco, si può arrivare all'espulsione, cioè alla privazione dei benefici del gioco. Si tratta di una conseguenza già accettata nell'atto di adesione all'impresa cooperativa sulla base del principio pacta sunt servanda. Nell'ambito strettamente giuridico si parla di invalidità delle azioni non conformi alle norme giuridiche, che vengono così private dei loro effetti giuridici. Come nota Bobbio, in tal caso non c'è l'uso della forza a svantaggio del trasgressore, ma la privazione dell'uso della forza a suo vantaggio ${ }^{71}$.

Le sanzioni privative in senso stretto punitive sono quelle che normalmente si ricollegano all'uso della forza fisica con modalità graduate, che culminano nella detenzione. Esse riguardano attività che sono necessarie per il mantenimento dell'ordine sociale e di una comunità politica stabile. Qui si manifesta con maggiore evidenza la connessione tra il diritto e la politica. Ma il diritto non si limita a regolare l'uso della forza, ma tende a restringerlo alle violazioni più gravi dei comportamenti sociali più basilari, limitando la tendenza espansiva contraria che è propria dello Stato. Insomma, il carcere dovrebbe essere l'extrema ratio da applicare nei confronti dei più gravi reati, che minacciano la stabilità e l'ordine sociale o offendono seriamente la libertà e la vita altrui, e da quelli compiuti da individui asociali, irrecuperabili o recidivi.

Le sanzioni costruttive sono di due tipi: quelle rieducative e quelle riparative. La rieducazione di chi ha compiuto azioni antisociali non è compatibile con la reclusione coatta, che è una forma di esclusione sociale, perché al contrario mira all'inclusione, cioè a sanare la rottura tra il reo e la società. Pertanto, la finalità rieducativa, che la costituzione italiana assegna alle pene, spinge ad un progressivo superamento dell'uso della forza fisica da parte del diritto.

71 BoвbIO, «Sanzione», pp. 115-117.

\section{$\odot \theta 00$}


È interessante notare che la rieducazione, come ogni forma di vera e propria educazione, esige la collaborazione del destinatario e che, quindi, è una forma di rispetto della sua agency. Le sanzioni riparative aggiungono alla finalità rieducativa la dimensione sociale del rapporto con la vittima, in quanto mirano alla riconciliazione con l'offeso come superamento del conflitto ${ }^{72}$. Possiamo anche chiamarle sanzioni relazionali, poiché focalizzano un rapporto sociale particolare a differenza di quelle rieducative che guardano all'intera società. Le sanzioni rieducative e quelle riparative sono un chiaro indice della possibilità di intendere la sanzione giuridica non più come una sostituzione della vendetta privata con la vendetta di Stato, ma come un percorso di continua ricucitura del tessuto sociale strappato.

Ovviamente questa è una tipologia astratta e sommaria, perché nella realtà queste differenti forme di sanzioni giuridiche si presentano spesso come intrecciate fra loro in modo a volte indistinguibile. Tuttavia la rilevanza delle sanzioni costruttive va chiaramente aumentando e quella delle sanzioni punitive diminuendo. L'obiezione che è improprio qualificare quelle costruttive come sanzioni vere e proprie è priva di rilevanza, poiché si tratta pur sempre di una risposta nei confronti della violazione della legge. Ciò che qui importa è la constatazione del disagio del diritto ad usare la violenza e la ricerca di forme di giustizia non violenta. Lo si capisce bene solo se si pensa all'eterna lotta del diritto nei confronti del dominio dell'uomo sull'uomo.

La teoria del diritto come pratica sociale è pienamente in grado di recepire e di giustificare questa evoluzione della sanzione giuridica, in quanto guarda il diritto non dall'alto del potere ma dal basso dell'inclusione e dell'integrazione sociale. Da questo punto di vista tale teoria assume anche una funzione evolutiva. Invece, se ci poniamo da un punto di vista descrittivo, non resta che riconoscere che il diritto è stato usato nei modi più diversi e per fini spesso inaccettabili. Dal punto di vista descrittivo non c'è alcun dover essere del diritto. Ma una pratica sociale, che anima un ordinamento normativo, porta in se stessa il suo dover essere, mirando a realizzarlo nel modo migliore possibile, anche se di fatto sempre con approssimazione.

72 Sul tema la bibliografia è già imponente. Rinvio soltanto a MANNOZZI, G.; LoDIGIANI, G.A. (a cura di), Giustizia riparativa. Ricostruire legami, ricostruire persone, Il Mulino, Bologna, 2015, in cui è da segnalare il contributo di Luciano Eusebi, che sottolinea nella giustizia riparativa la promozione della capacità di scelta e di autonomia delle persone, cosa pienamente in linea con la teoria del diritto come pratica sociale. 


\section{REFERENCIAS}

AGAZZI, E., «Per una riconduzione della razionalità tecnologica entro l'ambito della razionalità pratica», in Forme di razionalità pratica, a cura di S. Galvan, Franco Angeli, Milano, 1992, pp. 17-39.

AnDERSON, S.A., «The Enforcement Approach to Coercion», Fournal of Ethics \& Social Philosophy, 5 (2010), 1, pp. 1-31.

APEL, K.-O., Trasformation der Philosophie, Suhrkamp, Frankfurt, vol. I, 1973.

Austin, J., The Province of Jurisprudence Determined (1832), ed. by W.E. Rumble, Cambridge University Press, Cambridge, 1995.

Berman, M.N., «The Normative Functions of Coercion Claims», Legal Theory, 8 (2002), pp. 45-89.

Bоввіо, N., «Sanzione» (1969), ora in Contributi ad un dizionario giuridico, Giappichelli, Torino, 1994, pp. 307-333.

Boвbio, N., «Diritto e forza», ora in Studi per una teoria generale del diritto, a cura di T. Greco, Giappichelli, Torino, 2012, pp. 101-118.

Brady, P., «Coercion, Political Authority and the Common Good», The American fournal of Jurisprudence, 62 (2017), 1, pp. 75-87.

Bratman, M., Faces of Intention, Cambridge University Press, Cambridge, 1999.

Bustamante, T., «Coercion and the Normativity of Law: Some Critical Remarks on Frederick Schauer's The Force of Law», in The Force of Law Reaffirmed. Frederick Schauer Meets the Critics, eds. C. Bezemek and N. Ladavac, Springer, Switzerland, 2016, pp. 27-59.

Coleman, J., Practice of Principle. In Defence of a Pragmatist Approach to Legal Theory, Oxford University Press, Oxford, 2001.

Cover, R.M., «Nomos and Narrative», Harvard Law Review, 97 (1983), 1, pp. 4-68.

Coyle, S.; Pavlakos, G. (eds.), Furisprudence or Legal Science?, Hart Publishing, Oxford and Portland, 2005.

D’Agostino, F., La sanzione nell'esperienza giuridica, V ed., Giappichelli, Torino, 1999.

Dyzenhaus, D., «Law and Public Reason», McGill Law Journal, 38 (1993), pp. 366393.

Dworkin, R., Law's Empire, Fontana Press, London, 1986.

EndicotT, T., «The Reason of the Law», The American Fournal of Furisprudence, 48 (2003), pp. 83-106.

FINNIS, F., Natural Law \& Natural Rights, II ed., Oxford University Press, Oxford, 2011.

FISSEL, B.M., «Sanctions and Efficacy in Analytic Jurisprudence», Rutgers University Law Review, 69 (2017), pp. 1627-1652.

Fowler, M., «Coercion and Practical Reason», Social Theory and Practice, 8 (1982), 3, pp. 329-355.

FORST, R., The Right to Fustification, Columbia University Press, New York, 2007.

- Fustification and Critique. Towards a Critical Theory of Politics, Polity, Cambridge, 2013.

FULLER, L.L., The Morality of Law, revised edition, Yale University Press, New Haven and London, 1969. 
Giovanola, B., Giustizia sociale. Eguaglianza e rispetto nelle società diseguali, Il Mulino, Bologna, 2018.

GreEn, L., «The Force of Law: Duty, Coercion, and Power», Ratio furis, 29 (2016), 2, pp. 164-181.

Habermas, J., Teoria dell'agire comunicativo (1981), trad. it. di P. Rinaudo, vol. II, Il Mulino, Bologna, 1986.

HathaWaY, O.A.; SHAPIRO, S.J., «Outcasting: Enforcement in Domestic and International Law», The Yale Law fournal, 121 (2011), pp. 252-349.

Hiмmа, K.E., «Law and Coercion», in Encyclopedia of Law and Social Philosophy, eds. M. Sellers and S. Kirste, Springer, Dordrecht, 2017.

Kaufmann, A., Analogie und «Natur der Sache». Zugleich ein Beitrag zur Lebre vom Typus (1965), Decker \& C.F. Müller, Heidelberg, 1982.

Lagerspetz, E., «Obligations, Sanctions, and Coercion», in The Legal Ought, ed. by P. Chiassoni, Giappichelli, Torino, 2001, pp. 117-140.

LAMOND, G., «Coercion and the Nature of Law», Legal Theory, 7 (2001), pp. 35-57.

LEWIS, D., Convention: A Philosophical Study, Harvard University Press, Cambridge, 1969.

MacIntyre, A., After Virtue. A Study in Moral Theory, III ed., University of Notre Dame Press, Notre Dame (Indiana), 2007.

Mannozzi, G.; Lodigiani, G.A. (a cura di), Giustizia riparativa. Ricostruire legami, ricostruire persone, Il Mulino, Bologna, 2015.

NozIcK, R., «Coercion», in Philosophy, Science, and Method: Essays in Honor of Ernest Nagel, eds. S. Morgenbesser et al., St. Martin's Press, New York, 1969, pp. 440-472.

Pastore, B. et al., Le ragioni del diritto, Il Mulino, Bologna, 2017.

Pavlakos, G., Our Knowledge of the Law, Hart Publishing, Oxford and Portland, 2007.

PERrY, S., «Hart on Social Rules and the Foundations of Law: Liberating the Internal Point of View», Fordham Law Review, 75 (2006), 3, pp. 1171-1209.

Postema, G.J., «Implicit Law», Law and Philosophy, 13 (1994), pp. 361-387.

RAWLs, J., A Theory of Justice, revised edition, Oxford University Press, Oxford, 1999.

Raz, J., Practical Reason and Norms (1975), Princeton University Press, Princeton, 1990.

Ricoeur, P., Le Droit de punir, Intervention à la commission Nationale Justice et Aumônerie des Prisons sur «Le Sens de la peine», Paris, 2001.

Rodríguez-Blanco, V., Law and Authority Under the Guise of the Good, Hart Publishing, Oxford and Portland, 2014.

SCHAUER, F., The Force of Law, Harvard University Press, Cambridge, Mass., 2015.

SHAPIRO, S.J., «Law, Plan and Practical Reason», Legal Theory, 8 (2002), pp. 387-441.

Simmonds, N.E., «Law as an Idea We Live By», in The Cambridge Companion to Natural Law Furisprudence, eds. G. Duke and R.P. George, Cambridge University Press, New Yok, 2017, pp. 245-274.

Smith, M.N., «The Law as A Social Practice: Are Shared Activities at the Foundations of Law?», Legal Theory, 12 (2006), pp. 265-292.

VINX, L., Schauer on the Differentiation of Law, in The Force of Law Reaffirmed. Frederick Schauer Meets the Critics, eds. C. Bezemek and N. Ladavac, Springer, Switzerland, 2016, pp. 129-143. 
VIOLA, F., «Tre forme di positività del diritto», in Diritto positivo e positività del diritto, a cura di G. Zaccaria, Giappichelli, Torino, 1991, pp. 301-328.

— «La legalità come procedura e come prassi», Per la Filosofia, 10 (1993), n. 27, pp. 3043.

— «Pratiche sociali: istituzioni e procedure», Rivista di studi utopici, 2 (2007), 3, pp. 97106.

— «Il Rule of Law e il concetto di diritto», Ragion pratica, 30 (2008), pp. 151-168.

— Rule of Law. Il governo della legge ieri ed oggi, Giappichelli, Torino, 2011.

— «Il diritto tra arte ed etica», in Una filosofía del derecho en acción. Homenaje al profesor Andrés Ollero, coord. C. Hermida e J.A. Santos, Congreso de los Diputados, Madrid, 2015, pp. 3-17.

— «Una teoria deliberativa della giurisdizione?», Ars Interpretandi, 7 (2018), 1, pp. 1328.

— «Aquinas (On Natural Law)», in Encyclopedia of the Philosophy of Law and Social Philosophy, eds. M. Sellers and S. Kirste, Springer, Dordrecht, 2019.

VIOLA, F.; ZACCARIA, G., Diritto e interpretazione. Lineamenti di teoria ermeneutica del diritto, IX ed., Laterza, Roma-Bari, 2016.

Waldron, J., «Does Law Promise Justice?», Georgia State University Law Review, 17 (2000-2001), pp. 759-788.

— «The Concept and the Rule of Law», Georgia Law Review, 43 (2008), 1, pp. 1-61.

— «Self-application», NYU School of Law, Public Law Research, Paper N. 16-46, September 1, 2016. 